\title{
Synthesis of Optical Filters Using Sagnac Interferometer in Ring Resonator
}

\author{
Salvador Vargas, Member, IEEE, and Carmen Vázquez, Senior Member, IEEE
}

\begin{abstract}
We propose a new synthesis method for the implementation of infinite impulse response filters, using a Sagnac interferometer within a ring resonator. This structure permits the synthesis of second-order optical functions per stage. We present the parameters of the structure, which are related to the poles position, radial and azimuthal, and the design procedure, with an example of an autorregresive filter.
\end{abstract}

Index Terms-Optical filters, optical mirrors, Sagnac interferometers, synthesis, tuning.

\section{INTRODUCTION}

$\mathbf{T}$ HERE ARE many applications for photonics filters in modern optical communications systems. These applications include multiplexing/demultiplexing, equalization, dispersion compensation, dispersion slope compensation, and single sideband generation [1]. These photonics filters are synthesized using different methods as cascading all-pole and all-zero functions [2] by recurrence algorithm [3], [4], by appodization of coupling coefficients [5], [6], and closed forms derived from microwave counterpart [7].

Also we have seen recent interest in optical mirror ring resonator (OMRR)-based structures, that have been proposed as reflectors [8] or as tuneable filter, tuned by means of a coupling coefficient [9], [10].

But nobody has proposed a synthesis method that uses OMRR like building blocks for synthesis of infinite impulse response (IIR) filters. In this letter, a synthesis method based on OMRR, made of a Sagnac interferometer in a ring resonator (SIRR), as a second-order optical function is presented. The method is based on cascading all-pole and all-zero structures, but using an all-pole second-order structure (SIRR).

The main advantage of using the SIRR is that there is no need of phase shifters. It also has immunity to variations in the ring lengths. This is a key factor to produce the same time delay in a second-order transfer function realized with cascaded first-order transfer functions [2]. Finally, it has design flexibility, because this structure permits the synthesis of complex conjugated poles,

Manuscript received June 12, 2007; revised August 6, 2007. This work was supported in part by the Spanish CICYT (TEC2006-13273-C03-03-MIC), in part by the European project NoE EPhoton/One+, CAM (FACTOTEM-CM:S0505/ESP/000417), and in part by FENIS-CCG06-UC3M/TIC-0619.

S. Vargas was with the Universidad Carlos III de Madrid, Leganes, Spain. He is now with the Universidad Tecnológica de Panamá, Panamá 0819-07289 (e-mail: salvador.vargas@utp.ac.pa).

C. Vázquez is with the Universidad Carlos III de Madrid, Leganes 28911, Spain (e-mail: cvazquez@ing.uc3m.es).

Digital Object Identifier 10.1109/LPT.2007.908735

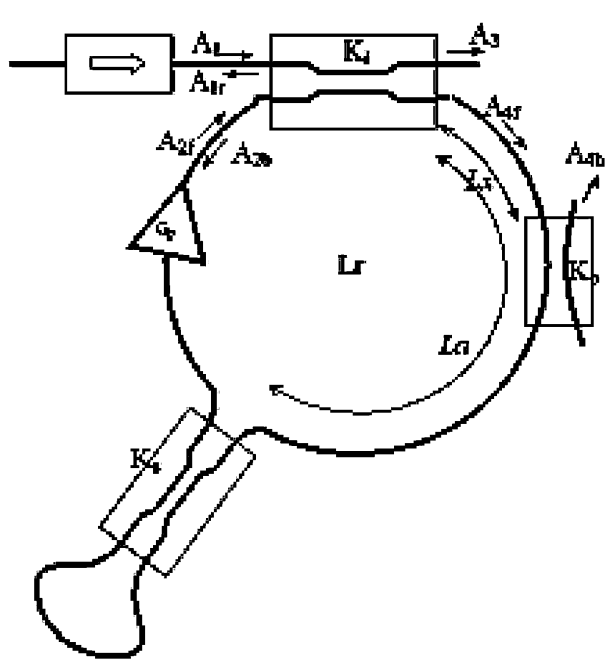

Fig. 1. Schematic of SIRR. Input $\left(K_{i}\right)$, output $\left(K_{o}\right)$, and Sagnac $\left(K_{s}\right)$ couplers. Amplifier $\left(G_{p}\right)$.

located in any place, by varying a coupling coefficient and an amplifier gain.

\section{STRUCTURE}

The SIRR is presented in Fig. 1. The effect of the Sagnac interferometer allows the double recirculation of light clockwise (forward) and counterclockwise (backward), in the ring. We assume that there is not mixing between these waves, because the coupling length is much greater than the wavelength of light, and that the couplers and the waveguides are lossless. The output transfer function of this structure is of second-order whatever point you take as output in the circuit. In Fig. 1, there is an isolator in the input port and an optical amplifier inside the ring. Both of them can be implemented in optical fiber technology, or in integrated optic technology [11], [12]. The first one is to prevent the wave reflected, as we are using each SIRR stage in the transmission mode, and the second one is used for giving design flexibility. There is also a variable coupler [13] in the Sagnac interferometer, like the amplifier, is for providing a reconfigurable design.

The output of the SIRR is taken from the coupler inside the ring, in the counterclockwise direction $\left(A_{4 b}\right)$ because this transfer function has two complex conjugated poles and zeroes only in the origin of the $Z$ plane. The unit time delay is given by the transit time of light in the ring length $(L r)$, which includes the Sagnac, the amplifier, and the couplers lengths.

The transfer function of the SIRR is derived using the transfer matrix formalism, as in [10, Appendix A], and is given by 


$$
\frac{A_{4 b}}{A_{1}}=\frac{-j 2 c_{i} c_{o} c_{s} d_{o} d_{s} z^{-\frac{2 L a-L x}{L r}}}{\left(1-Z p z^{-1}\right)\left(1-Z p^{*} z^{-1}\right)} .
$$

$c_{j}$ and $d_{j}$ are, respectively, the dimensionless coupling and transmitting coefficients of each coupler, where the sub-indexes $i, o$, and $s$ are input, output, and Sagnac couplers, respectively. $c_{j}$ and $d_{j}$ are related to the power coupling coefficient $K_{j}$ by means of $K_{j}=c_{j}^{2}$ and $1-K_{j}=d_{j}^{2}$.

$L a, L x$, and $L r$ are the length positions of the Sagnac coupler plus Sagnac length, output coupler, and ring length, respectively.

The poles $Z p$ given in (1) are complex conjugated and the modulus and phase of $Z p$ are given by

$$
\begin{aligned}
|Z p| & =d_{i} d_{o} G_{p}^{1 / 2} \\
\phi_{p} & =\arg \left(1-2 c_{s}^{2}+j 2 c_{s} d_{s}\right)
\end{aligned}
$$

where $G_{p}$ is the amplifier power gain.

The stability of this structure is guaranteed if the amplifier power gain $G_{p}$ is maintained less than $1 / \sqrt{ }\left(d_{i} d_{o}\right)$.

From (2), we see that the modulus of the poles is dependent on the gain and the power coupling coefficients of the input and output couplers. And from (3) it can be seen that the poles phases are only dependent on the power coupling coefficient of the Sagnac coupler. Both can be tuned independently. The best choice for tuning the radial position of the poles is using $G_{p}$, for avoiding problems when the pole is located near the unit circle, while for tuning the phases of the complex conjugated poles, $K_{s}$ is used. As both $G_{p}$ and $K_{s}$ can be variable, we can generate complex conjugated poles with any phase and modulus.

The value of $K_{s}$ from (3) generating the correct phases of the poles is given by

$$
K_{s}= \begin{cases}\frac{1}{2}-\frac{\left(\tan ^{2}\left(\left|\phi_{p}\right|\right)+1\right)^{-1 / 2}}{2}, & \left|\phi_{p}\right|<\pi / 2 \\ \frac{1}{2}+\frac{\left(\tan ^{2}\left(\left|\phi_{p}\right|\right)+1\right)^{-1 / 2}}{2}, & \left|\phi_{p}\right| \geqslant \pi / 2\end{cases}
$$

From (1), we see that this structure introduces some losses that can be minimized, once having fixed $K_{s}, G_{p}$, and $/ Z p /$, by maximizing the product $c_{i} \times c_{o} \times d_{o}$. This can be achieved using the power coupling coefficients of input and output couplers following:

$$
K_{i}=\frac{1-B}{1+B} ; \quad K_{o}=\frac{1-B}{2}
$$

with

$$
B=\frac{|Z p|^{2}}{G_{p}}
$$

\section{SYNTHESIS METHOD}

This synthesis method is applicable to the synthesis of IIR filters with real coefficients in their transfer functions. It is based

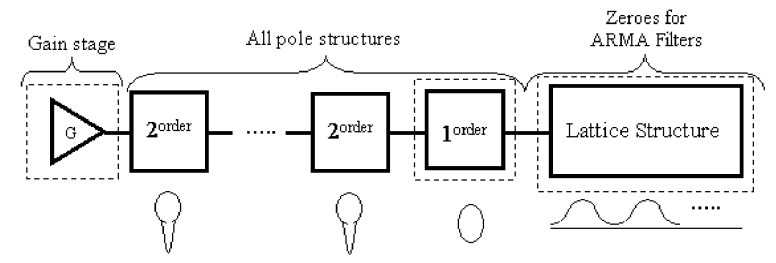

Fig. 2. Block diagram of synthesis structure. The structures in dashed lines are only needed in specific designs.

on cascading all-pole and all-zero structures, to generate autoregressive (AR) and autoregressive moving average (ARMA) filters. It uses the SIRR for generating an even number of complex conjugated poles, and adding a single pole optical function when the filter has an odd number of poles. For the synthesis of ARMA filters, the zeroes are implemented adding a stage of coherent two-port lattice delay line (TPLDL) circuits [3]. This is the most efficient structure for generating the zeroes in terms of number of components.

In Fig. 2, there is a block diagram of our filter, where we can see cascaded SIRR structures, the ring resonator for odd-order filters, and the lattice structure for ARMA filters. There is also an amplifier stage for compensating the losses introduced by the all-pole stages and the TPLDL circuit. The single-pole optical function used here is implemented by means of a ring resonator with gain, and a phase shifter, where output is taken inside the ring, using a coupler.

The transfer function of this device is given by

$$
\frac{A_{4}}{A_{1}}(z)=\frac{-c_{i r} c_{o r} z^{-\frac{L y}{L r}}}{1-Z p_{r} z^{-1}}
$$

where $c_{j}$ and $d_{j}$ are, respectively, the dimensionless coupling and transmitting coefficients of each coupler, where the sub-indexes $i r$ and or, represent input and output couplers, respectively, and $L y$ and $L r$ are the length to the output coupler position and the ring length, respectively. $Z p_{r}$ is the single pole of the transfer function, where modulus $\left(/ Z p_{r} /\right)$ and phase $\left(\phi_{p r}\right)$ are given by

$$
\begin{aligned}
\left|Z p_{r}\right| & =d_{i r} d_{o r} G_{p r}^{1 / 2} \\
\phi_{p r} & =-\varphi_{r}
\end{aligned}
$$

where $G_{p r}$ is the amplifier power gain, $\varphi_{r}$ is the phase change in the phase shifter.

From (7) and (8), we see that the pole phase is tuned by means of a phase shifter (adjusting the phase shifter to the negative of the pole phase), and the modulus by means of the amplifier gain $G_{p r}$. The gain is used for tuning the modulus giving design flexibility and to avoid the effect of waveguide and coupler losses here neglected. The losses induced by this stage once $G_{p r}$ and $/ Z p_{r} /$ are fixed, can be minimized by taking input and output coupler coefficients given by

$$
K_{i r}=K_{o r}=1-\frac{\left|Z p_{r}\right|}{G_{p r}^{1 / 2}}
$$



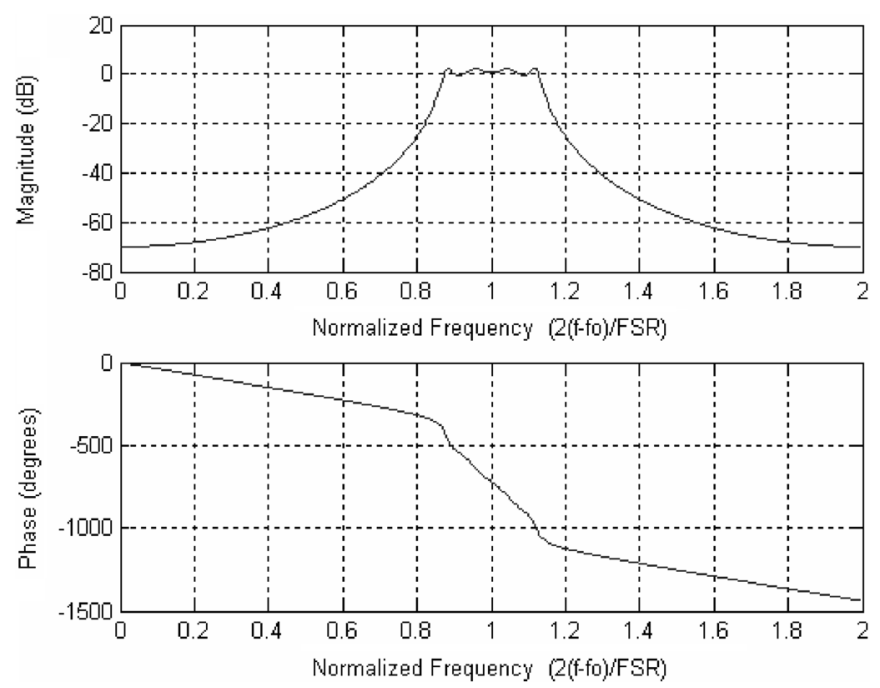

Fig. 3. Frequency response of fourth-order filter. $f o$ is the central frequency and FSR, the free-spectral range.

TABLE I

Modulus ANd Phase of the FILTER POLES

\begin{tabular}{ccc}
\hline \hline Poles & $/ \mathrm{Zp} /$ & $\phi_{\mathrm{p}}(\mathrm{rad})$ \\
\hline$-0.8936+\mathrm{j} 0.1310$ & 0.9032 & 2.996 \\
$-0.8936-\mathrm{j} 0.1310$ & 0.9032 & -2.996 \\
$-0.8935+\mathrm{j} 0.3585$ & 0.9627 & 2.760 \\
$-0.8935-\mathrm{j} 0.3585$ & 0.9627 & -2.760 \\
$\mathrm{Z \textrm {p }} /=$ poles modulus and $\phi_{\mathrm{p}}=$ poles phases. & \\
\hline \hline
\end{tabular}

These values of power coupling coefficients provide the minimum losses and the correct radial position of the pole.

\section{EXAMPLE AND DISCUSSION}

For a detailed explanation, an example of the synthesis of an IIR filter is reported. Its frequency response is shown in Fig. 3, and its $Z$ transfer function is given by

$$
H[z]=\frac{0.0043 z^{-4}}{1+3.5742 z^{-1}+4.9363 z^{-2}+3.1142 z^{-3}+0.7560 z^{-4}} .
$$

The filter has a full-width at half-maximum of 0.13 times the free-spectral range, with less than $2 \mathrm{~dB}$ of ripple and a crosstalk of $70 \mathrm{~dB}$. This is a fourth-order AR filter having real coefficients, so two SIRR stages are needed for implementation.

The modulus and phase of the filter poles are shown in Table I. Knowing the phase poles $\left(\phi_{p}\right)$ and using (4) we can find the values of $K_{s}$. Fixing $G_{p}$ to 2 , in both stages, knowing $/ Z p /$ and using (5), we can find $K_{i}$ and $K_{o}$ for each SIRR stage.

The obtained values following this procedure are shown in Table II. The losses introduced by the cascaded SIRR are 0.0043 , and this means that we will not need the gain stage.
TABLE II

Resulting Parameters of the SIRR Per Stage

\begin{tabular}{ccccc}
\hline \hline Stage & $\mathrm{G}_{\mathrm{p}}$ & $\mathrm{K}_{\mathrm{s}}$ & $\mathrm{K}_{\mathrm{i}}$ & $\mathrm{K}_{\mathrm{o}}$ \\
\hline 1 & 2 & 0.995 & 0.421 & 0.296 \\
2 & 2 & 0.964 & 0.367 & 0.268
\end{tabular}

$\mathrm{K}_{\mathrm{i}}, \mathrm{K}_{\mathrm{o}}$, and $\mathrm{K}_{\mathrm{s}}$ are the power coupling coefficients of the in, out and Sagnac couplers and $\mathrm{G}_{\mathrm{p}}$ is the amplifier power gain, of each SIRR stage.

\section{CONCLUSION}

A novel synthesis method based on a cascading all-pole transfer function based on a ring resonator with an internal Sagnac loop (SIRR) has been reported. In this method, there is no need of phase shifters in each stage to properly locate poles on the $z$ plane, but uses variable coupling coefficients and gain. There is also no influence of length tolerances in complex conjugated poles implementation. Closed-form formulas describing the synthesis process are given. A specific example of a fourth-order AR filter, with less than $2 \mathrm{~dB}$ of ripple and a crosstalk of $70 \mathrm{~dB}$ is reported for describing the method. State-of-the-art technology in optical fiber an integrated optics is available for building filters using this method.

\section{REFERENCES}

[1] A. Meijerink et al., "Phased array antenna steering using a ring resonator-based optical beam forming network," in Commun. Vehicular Technology, 2006 Symp., Nov. 2006, pp. 7-12.

[2] N. Q. Ngo and L. N. Binh, "Novel realization of monotonic butterworth-type lowpass, highpass, and bandpass optical filters using phasemodulated fiber-optic interferometers and ring resonators," J. Lightw. Technol., vol. 12, no. 5, pp. 827-841, May. 1994.

[3] K. Jinguji and M. Kawachi, "Synthesis of coherent two-port latticeform optical delay-line circuit," J. Lightw. Technol., vol. 13, no. 1, pp. 73-82, Jan. 1995.

[4] K. Jinguji, "Synthesis of coherent two-port optical delay-line circuit with ring waveguides," J. Lightw. Technol., vol. 14, no. 8, pp. 1882-1898, Aug. 1996.

[5] M. Kuznetsov, "Cascaded coupler mach-zehnder channel dropping filters for wavelength-division-multiplexed optical systems," J. Lightw. Technol., vol. 12, no. 2, pp. 226-230, Feb. 1994.

[6] M. Sharma, H. Ibe, and T. Ozeki, "Optical lattice-type add-drop multiplexing filters and their use in WDM networks," Opt. Fiber Technol., vol. 4, no. 1, pp. 117-134, Jan. 1998.

[7] A. Melloni and M. Martinelli, "Synthesis of direct-coupled-resonators bandpass filters for WDM systems," J. Lightw. Technol., vol. 20, no. 2, pp. 296-303, Feb. 2002

[8] O. Schwelb, "Band-limited optical mirror based on ring resonators: Analysis and design," J. Lightw. Technol, vol. 23, no. 11, pp. 3931-3946, Nov. 2005

[9] C. Vazquez, S. Vargas, J. M. S. Pena, and P. Corredera, "Tunable optical filters using compound ring resonators for DWDM," IEEE Photon. Technol. Lett., vol. 15, no. 8, pp. 1085-1087, Aug. 2003.

[10] C. Vázquez, S. E. Vargas, and J. M. Sánchez-Pena, "Sagnac loop in ring resonators for tunable optical filters," J. Lightw. Technol., vol. 23 no. 8, pp. 2555-2567, Aug. 2005.

[11] J. S. Yang, J. W. Roh, S. H. Ok, D. H. Woo, Y. T. Byun, W. Y. Lee, T. Mizumoto, and S. Lee, "An integrated optical waveguide isolator based on multimode interference by wafer direct bonding," IEEE Trans. Magn., vol. 41, no. 10, pp. 3520-3522, Oct. 2005.

[12] V. M. Menon, V. Tong, and S. R. Forrest, "Control of quality factor and critical coupling in microring resonators through integration of a semicondutor optical amplifier," IEEE Photon. Technol. Lett., vol. 16, no. 5, pp. 1343-1345, May 2004.

[13] J. Yao, D. Leuenberger, M. Lee, and M. C. Wu, "Silicon microtoroidal resonators with integrated MEMS tunable coupler," IEEE J. Sel. Topics Quantum Electron., vol. 13, no. 2, pp. 202-208, Mar./Apr. 2007. 